\title{
How do diquark fluctuations and chiral soft modes affect di-lepton production in the deconfined phase
}

\section{Teiji Kunihiro*}

Yukawa Institute for Theoretical Physics, Kyoto University, Kyoto 606-8502, Japan

E-mail: kunihiro@yukawa.kyoto-u.ac.jp

\section{Masakiyo Kitazawa}

Department of Physics, Osaka University, Toyonaka, Osaka 560-0043, Japan

E-mail: masky@yukawa.kyoto-u.ac.jp

\section{Yukio Nemoto}

Department of Physics, Nagoya University, Nagoya 464-8602, Japan

E-mail: nemoto@hken.phys.nagoya-u.ac.jp

We examine diquark fluctuations and chiral soft modes existing in the precritical region of color superconductivity and chiral transition, respectively, at finite temperature and density. We evaluate how they contribute to anomalous di-lepton production; although there appear peaks in the spectral function owing to the existence of the soft modes, the enhancement of the production rate may not be so prominent to be a clear signal of the phsase transitions.

Critical Point and Onset of Deconfinement 4th International Workshop

July 9-13 2007

GSI Darmstadt,Germany

\footnotetext{
* Speaker.
} 

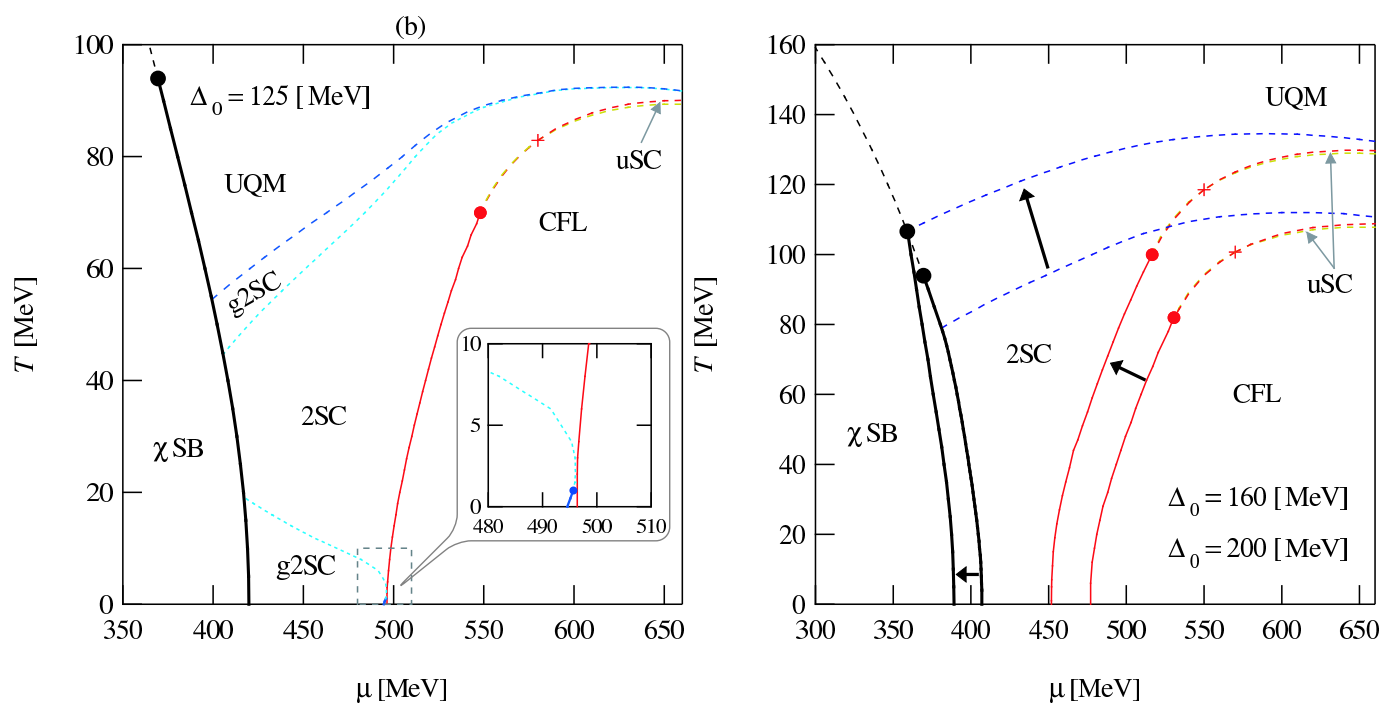

Figure 1: Left panel; the phase diagram for the intermediate strength of the pairing interaction for the three flavor $(q=u, d, s)$ case under the charge and color neutrality constraint. Right panel; the phase diagram for the large strength of the pairing interaction. Others are the same as the left panel. Taken from [1]; see this reference for the details.

\section{Introduction}

In this report, we focus on the precursory phenomena of two of QCD phase transition at finite temperature and/or density, i.e., the chiral transition and the color superconductivity. We assume that the phase transitions are almost of second order and hence specific soft modes exist around the respective critical points.

\section{Precursory phenomena of color superconductivity in heated quark matter}

In this part, we shall consider quark matter in the normal phase but near the critical temperature $T_{c}$ of the color superconductivity and discuss the possibility to observe pre-critical phenomena of color superconductivity by the heavy-ion collisions.

Owing to the many internal degrees of freedom of quarks, there are rich varieties of the pairing patterns of the color superconductivity. However, the highest- $T$ phase which is relevant for heavyion collisions at high baryon densities is the two-flavor superconducting (2SC) phase[1]. We show examples for the 2SC phase boundaries obtained in Nambu-Jona-Lasinio-type models in Fig. 1.

We explore the possibility to see precursory phenomena of color superconductivity in the quark matter possibly created by the heavy-ion collisions. Since we are interested in the high- $T$ phase because heavy-ion collisions can only create heated matter, we may thus focus on the 2SC phase. Our discussions are based on the observation that there can exist a rather wide pre-critical region of the color superconductivity in the $T$ - $\mu$ plane at moderate density[2], as shown in Fig. 2, where the small pairing coupling is taken, and the neutrality constraints are not imposed. The large diquark-pair fluctuations may affect various observables leading to precursory phenomena[2, 3]. 


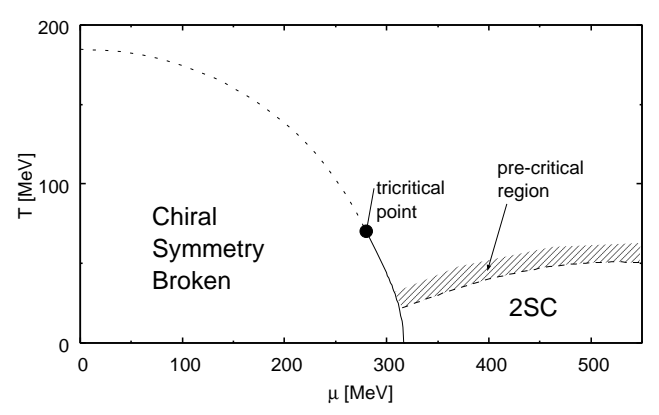

Figure 2: Phase diagram for isospin-symmetric two-flavor quark matter from an NJL-type model with a relatively weak coupling [4]. The critical temperatures for the $2 \mathrm{SC}$ phase transition is model-dependent, as seen from the comparison with Fig.1.

In the extremely high-density region, the perturbative calculation should be valid and the phase transition to the color-superconducting phase is a first order due to the gauge fluctuations in this region. On the other hand, the color superconductivity is expected to turn to a type-II at lower density[5]. We shall only consider the effects of the pair fluctuations at moderate density which is relevant to heavy-ion collisions.

We first notice that the ratio of the diquark coherence length to the average inter-quark length, which is proportional to $E_{F} / \Delta$ with $E_{F}$ being the Fermi energy, can be as small as $\sim 10$ at moderate densities. The stronger the interaction between the quarks, the shorter the coherence length, which can be as small as almost the same order of the inter-quark distance[6, 7]. The short coherence length implies that the fluctuation of the pair field is significant and the mean-field approximation looses its validity.

The large fluctuations cause an excess of the specific heat, which eventually diverges at $T_{c}$ owing to the critical fluctuations for the second order transition[4], as shown in Fig.3: We can see that as the temperature approaches $T_{c}$ from above, the anomalous part of the specific heat $c_{\mathrm{v}}^{\mathrm{fl}}$ become significant. Such an anomalous increase of the specific heat may affect the cooling of the proto-compact stars. Here one should, however, notice that the critical divergence of the specific heat is essentially due to the static fluctuations of the pair field[8]; the temperature region where static fluctuations is significant $[9,4]$.

We now turn to the discussions on the dynamical fluctuations of the diquark pair field. At finite temperature, the dynamical fluctuations of the pair field become also significant and develop a well-defined collective mode as the temperature is lowered toward $T_{c}$ if the color superconducting phase transition is of second order[2]: The spectral function of the diquark fluctuations gets to have a sharp peak in the low-energy region at about $T=1.2 T_{c}$, and the peak position decreases as the temperature is lowered toward the critical temperature. This collective soft mode is found to be a diffusive mode.

The soft mode of the color superconductivity in the heated quark matter leads to the formation of a pseudogap in the density of states (DOS) of quarks, i.e., an anomalous depression in the DOS around the Fermi surface[3, 4]; the quark propagator is modified due to the coupling with the fluctuating pair-field or the pairing soft mode, as shown in Fig. 4. 


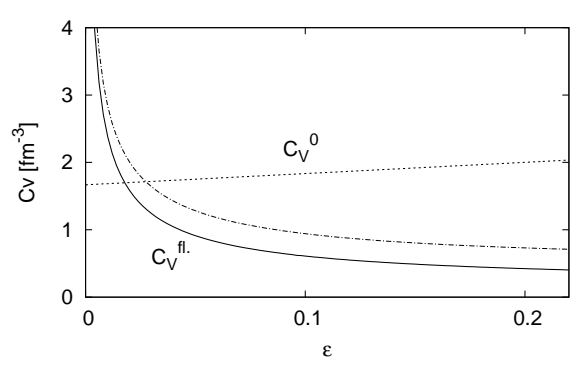

Figure 3: The specific heat per unit volume $c_{v}^{\text {fl. }}$ with the effects of the fluctuations of the pair field, together with that in the free fermionic system, $c_{\mathrm{v}}^{0}$. The total specific heat is given by $c_{\mathrm{v}}=c_{\mathrm{v}}^{0}+c_{\mathrm{v}}^{\mathrm{fl}}$. The dot-dashed curve represents the specific heat obtained with only the static part of $\Omega_{\mathrm{fl} .} . \varepsilon \equiv\left(T-T_{c}\right) / T_{c}$.

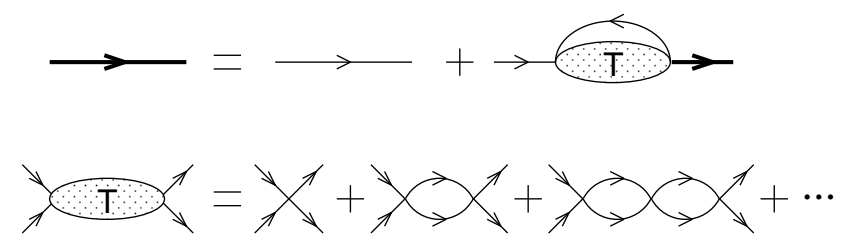

Figure 4: The Feynman diagrams representing the quark Green function in the T-matrix approximation employed in Ref. [4]. The thin (bold) lines represent the free (full) propagator and the wavy line denotes the pairing soft mode.

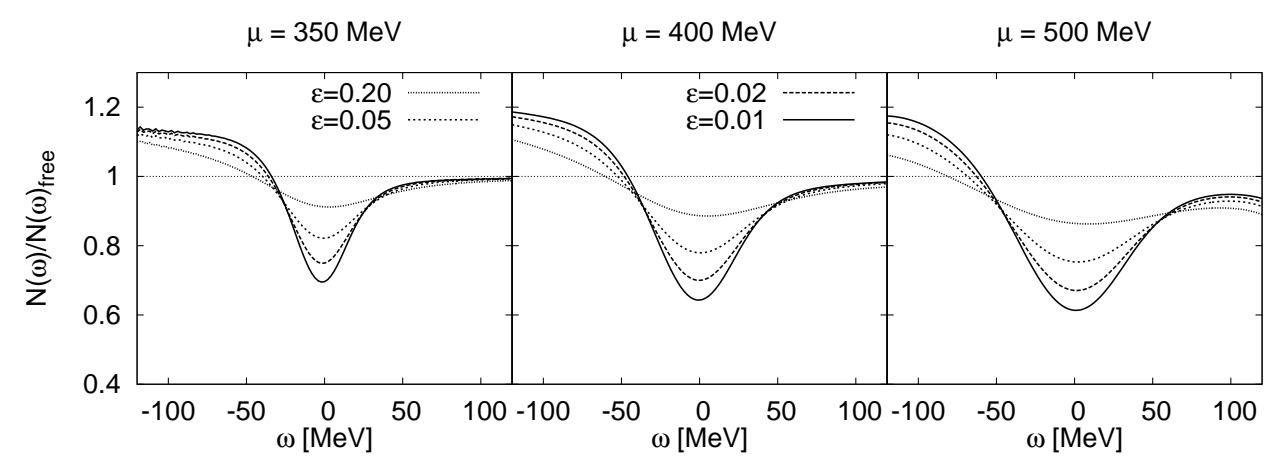

Figure 5: Density of states for quark matter near but above $T_{c}$ with several reduced temperatures $\varepsilon$ and quark chemical potentials $\mu=350,400,500 \mathrm{MeV}$ [3]. One sees a clear pseudogap phenomenon irrespective of $\mu$ near $T_{c}$.

As shown in Fig. 5, there appears a depression in the DOS around the Fermi energy for each $\mu$ near $T_{c}$, and they survive up to $\varepsilon \equiv\left(T-T_{c}\right) / T_{c} \approx 0.1$ irrespective of $\mu[3,4]$. This is an incomplete gap, or "pseudogap" formed within the QGP phase above $T_{c}$. The pseudogap in the DOS implies that the quarks around the Fermi surface have a short life time owing to the decay process $\mathrm{q} \rightarrow$ hole $+(\mathrm{qq})_{\text {soft }}$ emitting the soft mode like Cherenkov process. This short-livedness of the quarks around the Fermi surface means that the system is not a Fermi liquid [4, 12]. We notice that the pseudogap formation is known as a characteristic behavior of the materials which become the 

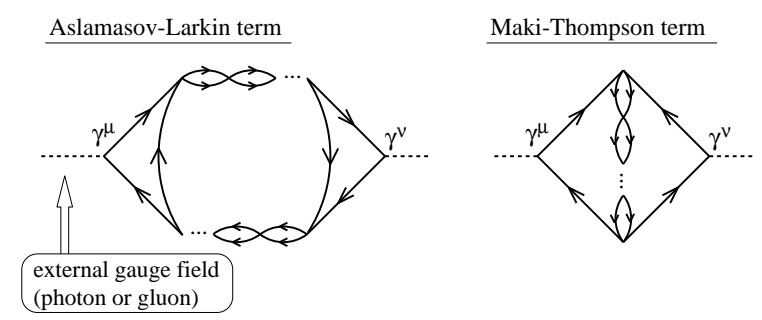

Figure 6: The diagrams that contribute to the photon (or gluon) self-energy representing the AslamazovLarkin (left) and the Maki-Thompson (right) terms[4]. The wavy lines denote the soft mode.

high- $T_{c}$ superconductors(HTSC)[11, 10]. Thus we can say that the heated quark mater at moderate densities is similar to the HTSC materials rather than the usual superconductors of metals.

It is known that pair fluctuations above $T_{c}$ cause a large excess of the electric conductivity, which is called the paraconductivity in condensed matter physics. Two microscopic mechanisms that give rise to such an anomalous conductivity are identified in terms of Feynman diagrams. They are called Aslamazov-Larkin (AL) and Maki-Thompson terms[13], both of which are depicted in Fig. 2; the dotted lines in the figure denote the gauge field, i.e., the photon in this case. The colorconductivity would be also enhanced by the similar mechanisms near $T_{c}$, although it would be of academic interest only. Our point is that the photon self-energy $\Pi^{\mu v}(Q)$ in the quark matter at $T>T_{c}$ can be also modified due to the fluctuations of the diquark pair-field as well as inside the color superconducting phase[16]: The diagrams shown in Fig. 2 can be interpreted as modifications of the self-energy of the gauge fields, i.e., $\Pi^{\mu v}(Q)=\mathscr{F}\left[i \theta(t)\left\langle\left[j^{\mu}(x), j^{v}(0)\right\rangle\right]\right.$, where $\mathscr{F}$ denotes the Fourier transformation. The external photon field thus can couple to the soft mode of the color superconductivity with the pairing soft mode in the diagrams being replaced by the diquark pair fields. This is interesting, because modifications of the photon self-energy may be detected as an enhancement of the invariant-mass distribution of the dileptons emitted from the created matter. The lepton-pair production rate per four-momenta is given by the well-known formula[14]

$$
\frac{d \Gamma}{d^{4} Q}=\frac{-\alpha g^{\mu v} \operatorname{Im} \Pi_{\mu v}(Q)}{12 \pi^{4} Q^{2}\left(\mathrm{e}^{\beta q_{0}}-1\right)} .
$$

The more direct observable in experiment is the invariant mass spectrum of dilepton production rate, which is given by integrating $d \Gamma / d^{4} Q$ over four momenta

$$
\frac{d \Gamma}{d M^{2}}=\int \frac{d^{3} q}{2 q^{0}} \frac{d \Gamma}{d^{4} Q} .
$$

We show in Fig. 7 a preliminary result[15] of $d \Gamma / d M^{2}$ obtained from the $\Pi_{\mu v}(Q)$ with the $\mathrm{Al}$ term, which is evaluated in some approximation[15]: The solid lines denote the production rate with the AL term for some reduced temperatures $\varepsilon \equiv\left(T-T_{c}\right) / T_{c}$ at $\mu=400 \mathrm{MeV}$, while the dashed lines show the production rate from the free quark system for $T=T_{c}$ and $1.5 T_{c}$ at $\mu=400 \mathrm{MeV}$. The Figure shows that the contribution of the AL term causes a large enhancement with a sharppeak structure in the production rate in the lower energy region, and the peak becomes larger and sharper as the temperature approaches $T_{c}$. It is known that a similar behavior of $d \Gamma / d M^{2}$ is also 


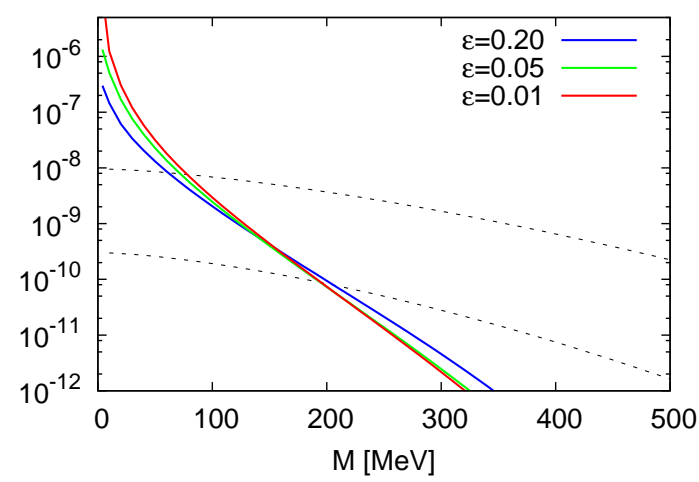

Figure 7: A preliminary result for the dilepton production rates Eq.(2.2) for several reduced temperatures $\varepsilon \equiv\left(T-T_{c}\right) / T_{c}$ near but above $T_{c}$ with $\mu=400 \mathrm{MeV}[15]$. The solid lines include the contributions of the Aslamazov-Larkin term, while the dashed lines represent the dilepton rate from the free quark matter at $T=T_{c}$ (below) and $T=1.5 T_{c}$ (above).

seen in the color superconducting phase itself[16]. In both cases, the characteristic enhancement of $d \Gamma / d M^{2}$ appears at energies less than the pion mass. Therefore, experimental observations of the dilepton production rate at low energy might be able to detect an signature of a formation or a precursor of the color superconductivity in the heavy-ion collisions. Such a low-mass energy region is, however, also the region where other hadronic and electro-magnetic process contribute to the dilepton production. So a dis-entanglement from these processes have to be made to identify the AL process due to the precursory diquark pairing fluctuations, which might be, unfortunately, difficult to perform. One should estimate also the contribution from the Maki-Tompson term as well as confirm the present estimate of the AL term more definitely.

\section{The soft modes of chiral transition and enhancement of lepton-pair production}

The chiral transition is a QCD phase transition with the order parameter $\langle\bar{q} q\rangle$. If the phase transition is of second order, of weak first order or of cross over, there should be a critical region where the chiral fluctuations $\left\langle(\bar{q} q)^{2}\right\rangle$ and $\left\langle\left(\bar{q} i \gamma_{5} \tau_{a} q\right)^{2}\right\rangle$ in the scalar and pseudoscalar channels, respectively, significant. It means that there will exist elementary soft modes or quasi-particles corresponding to these fluctuations in the critical region[17]. In the following discussions, we will assume that the chiral transition at finite temperature and/or density has a critical region where such chiral soft modes have significant strength. In this context, it is noteworthy that the lattice people determine the critical temperature of the chiral transition from the peak position of the chiral susceptibility in the scalar channel, i.e., $\chi_{m} \equiv \partial / \partial m\langle\langle\bar{q} q\rangle\rangle=\left\langle\left\langle(\bar{q} q)^{2}\right\rangle\right\rangle$; which surely shows a peak behavior around some temperature[18]. In other words, the so called generalized mass squared defined by $m_{\sigma}^{2} \equiv \chi_{m}^{-1}$ decreases as $T$ goes high and gives the minimum around the critical temperature and then increases in the chirally symmetric phase; in the high $T$ phase, the generalized masses in the $\sigma$ and the $\pi$ channels tend to be degenerated.

Recently, people are much interested in the properties of the QGP phase near the critical temperature $\left(T_{c}\right)$ [19]: The success of the perfect hydrodynamics in reproducing the elliptic flow 
of hadrons in the heavy-ion collisions at the Relativistic Heavy Ion Collider (RHIC) suggests that the created matter is a strongly coupled system. One of the interesting problems on the QGP phase near $T_{c}$ is the possible existence of hadronic excitations even in the QGP phase near $T_{c}[17,20]$. The existence of the mesonic excitations in the light-quark sector was suggested as being the soft modes associated to the chiral transition [17]: The spectral function calculated in the NJL model shows the existence of degenerated hadronic soft modes called 'para-pion' and 'para-sigma' at $T>T_{c}$. It was found that the soft modes acquire a large strength but with small width as $T$ approaches $T_{c}$ from above; it means that the soft modes become a good elementary modes in the vicinity of $T_{c}$.

\subsection{QCD phase transition at finite $T$ and $\mu$ with finite quark mass}

In the chiral limit with two flavors $(q=u, d)$, the chiral transition at finite temperature with vanishing chemical potential $\mu$ is known to be second order[21]: As $\mu$ is raised, the critical line of the second-order transition extends up to some critical point $\mathrm{P}_{\mathbf{T C P}}:\left(T_{\mathbf{T C P}}, \mu_{\mathbf{T C P}}\right)$ in the $(T, \mu)$-plane. The point $\mathrm{P}_{\mathbf{T C P}}$ is a tri-critical point because from it the critical line turns to a critical line of the first-order transition for higher density and lower temperatures. When the current quark masses are introduced, the second-order transition at small $\mu$ region changes to a cross-over[21, 22]. The common belief based on calculations of some chiral effective models[23] is that the critical line of the first-order transition in the large $\mu$ region remains; the tricritical point changes its nature to a critical end point $\mathbf{P}_{\mathbf{C E P}}$.

It should be noticed, however, that there is some caveats to this belief that the QCD phase transition at small temperature is of first order. It has been known for some time [21] that the inclusion of the vector term $G_{V}\left(\bar{q} \gamma_{\mu} q\right)^{2}$ can drastically alter the nature of the chiral transition at small temperature but at finite density where the color superconductivity was not taken into account; with an increase of the coupling constant $G_{V}$, the position of $\mathrm{P}_{\mathbf{C E P}}$ moves to lower $T$ and higher $\mu$, and eventually disappear in the $(T, \mu)$-plane! When the color superconductivity is incorporated, more interesting phenomenon can occur[24] as shown in Fig. 8: It is noteworthy that there appear another critical end point in the lower $T$, and hence there can exist two end points at both sides of the critical line of the first-order transition for the vector coupling $G_{V} / G_{S}=0.35$ where the $G_{S}$ denotes the strength of the scalar coupling $(\bar{q} q)^{2}$ in the NJL model. We find that the coexisting-CSC transition at low temperatures becomes a crossover transition, and there exists a coexisting phase where both the chiral and the diquark condensates have finite values. For larger $G_{V}$, the critical line for the first-order transition disappears completely and there exists only the cross-over in the entire $(T, \mu)$ plane. It is interesting that such a coexisting phase of the chirally-broken and color superconducting phase and hence the appearance of the new critical end point has been advocated where the driving force of the coexistence is not due to the vector coupling but the axial anomaly term[25].

\subsection{The soft modes around the critical end point}

The phase transition at the critical end point $P_{\mathbf{C E P}}$ is of second order. Then what is the soft mode for this second order transition? The $\sigma$ meson as the fluctuation of the chiral condensate has still a non-zero mass at $P_{\mathbf{C E P}}$ because the chiral symmetry is explicitly broken. Fujii[26] showed that the soft mode is a hydrodynamical mode which has the strength in the space-like region of the momentum-energy plane: At finite density, the charge conjugation symmetry is broken and the 

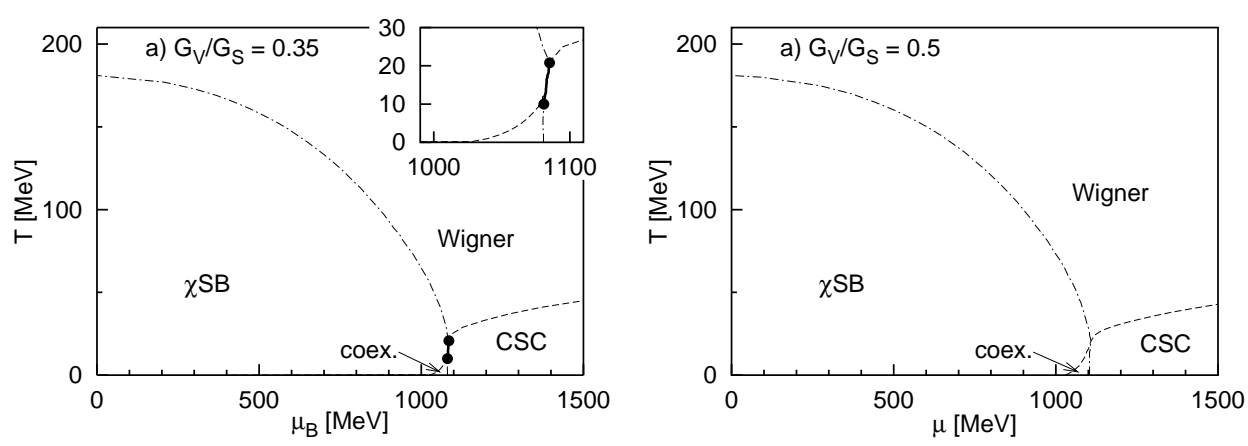

Figure 8: The left panel: The phase diagram with $G_{V} / G_{S}=0.35$ in the $T-\mu$ plane. There appear two endpoints of the first-order transition. The right panel: The phase diagram in the $T-\mu$ plane with $G_{V} / V_{S}=$ 0.5 . The critical line for the first-order transition disappears completely and the critical end point disappears with the large vector coupling.

scalar-vector mixing can occur and the soft mode in this case consists of the density fluctuation and the chiral fluctuation, of which the former is the main component of the mode. Since this soft mode exists in the space-like region, it can not affect particle productions to be seen in the timelike region. It can, however, contribute to the hydrodynamical modes, leading to dynamical critical phenomena[27].

\subsection{Di-lepton production owing to the scalar-vector mixing}

Although the sigma-mesonic mode is not a genuine soft mode around $\mathrm{P}_{\mathbf{C E P}}$, it can show a slight softening as the system approaches the critical temperature from above. So we shall examine effects of the sigma mesonic mode in the time-like region on the lepton-pair production[28]. The relevance of the scalar mode to the photon properties in the system can be understood as follows. As we mentioned in the previous subsection, there arises the scalar-vector mixing at finite $\mu$; the zero-th component of the vector is relevant and it is the baryon density. The importance of such a mixing was noticed in the work on the baryon-number susceptibility in association of the chiral transition[29]; it was first shown that the quark-number susceptibility can be enhanced around the critical point of the chiral transition at finite density as well as finite temperature.

The baryon-number susceptibility is a static quantity. The scalar-vector mixing also occurs in the dynamical cases. Owing to the mixing, the photon self-energy can be affected by the possible change of the $\sigma$ mode mass.

We calculate the invariant mass distribution of electron pairs using the formula given in Eq.2.1) where the photon self-energy include the contribution from the soft mode as given in Fig.9 as well as the bare one given in Fig.10. The resultant electron-pair production rate is shown in the left panel of Fig11, where the temperature dependence of the production rate is shown; The upper curves correspond to higher temperature away from $T_{c}$. We can see an enhancement of the production, which is encouraging but unfortunately may not be sufficient for detecting in experiments. Here it should be noted that the figure shows the invariant mass distribution of the lepton pair where the out-coming momenta are integrated out. However, the momentum-non-integrated probability show a clear peak, although not shown here. 


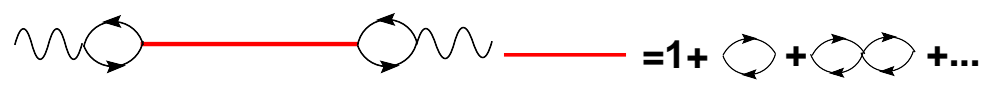

Figure 9: The left graph shows the anomalous photon self-energy owing to the chiral soft mode denoted by the straight line in the middle. The straight line is actually composed of the scalar bubble diagrams as shown in the right panel.

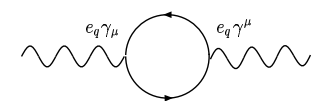

Figure 10: The bare self-energy diagram of the photon due to the quark loop.
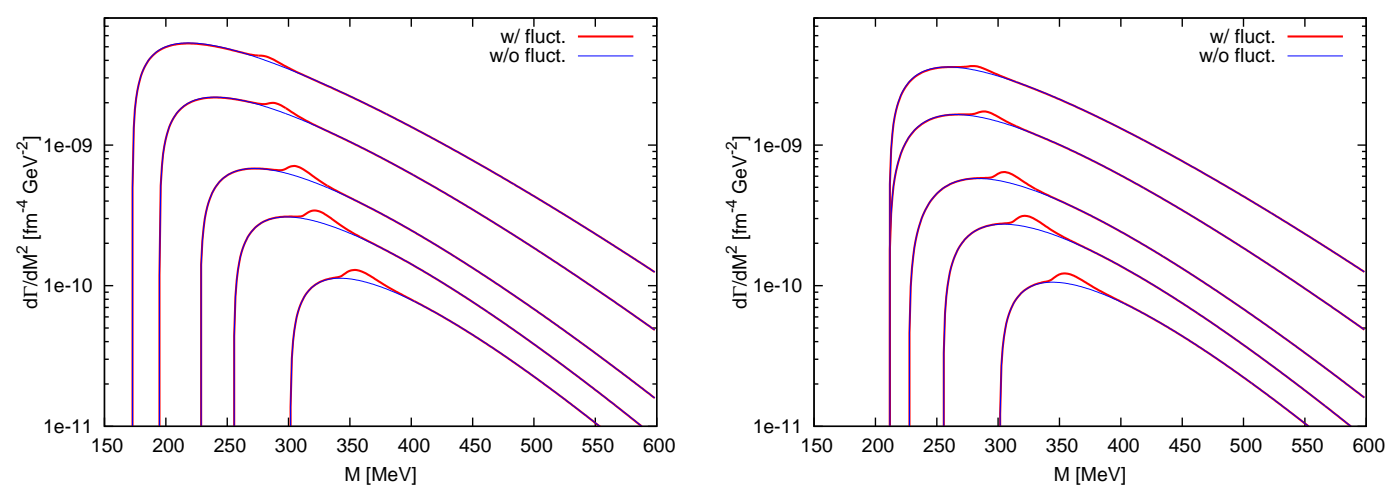

Figure 11: Left panel: the di-electron production rate for temperatures, $1.3 T_{c}, 1.2 T_{c}, 1.1 T_{c}, 1.05 T_{c}$ and $1.01 T_{c}$; the upper curves correspond to the higher temperatures. Right panel: the di-muon production rate. Others are the same as the left panel.

The right panel of Fig.11 shows the result for the muon-pair production, where the fourmomenta dependent production rate is given by

$$
\frac{d \Gamma}{d^{4} Q}=\frac{-\alpha g^{\mu v} \operatorname{Im} \Pi_{\mu v}(Q)}{12 \pi^{4} Q^{2}\left(\mathrm{e}^{\beta q_{0}}-1\right)} \cdot\left[1+\frac{2 m_{\mu}^{2}}{q^{2}}\right]\left[1-\frac{4 m_{\mu}^{2}}{q^{2}}\right]^{1 / 2}
$$

instead of Eq.(2.1).

One sees that some enhancement of the muon-pair production rate over the normal one as in the case of the lepton-pair production. However, the enhancement may not be sufficient to detect by experiments.

\section{Summary and concluding remarks}

We have first emphasized the importance of the notion of the soft modes of QCD phase transitions at temperatures above $T_{c}$; they may be hadronic excitations above $T_{c}$. We have discussed the two QCD phase transitions, i.e., the color superconductivity and the chiral transition. The soft modes of the so called 2SC of the color superconductivity can cause an enhancement of the electron-pair production in rather low invariant mass region. We have noticed that the soft mode 
around the critical end point where the transition is second order is a kind of density fluctuations coupled with the $\sigma$-meson like fluctuations. This soft modes have the dispersion relation which is space-like. Nevertheless, we explored how the sigma mesonic modes in the time-like region affect the di-lepton productions; the violation of the charge conjugation at finite $\mu$ is responsible for the fact that the sigma mode can affect to the photon self-energy in the hot medium with the non-vanishing $\mu$.

\section{Acknowledgements}

T. K. is supported by a Grant-in-Aid for Scientific Research by Monbu-Kagakusyo of Japan (No. 17540250).

M. K. is supported by a Grant-in-Aid for Scientific Research by Monbu-Kagakusyo of Japan (No. 19840037). Y. N. is supported by a JSPS Grant-in-Aid for Scientific Research (\#18740140). This work is supported by the Grant-in-Aid for the 21st Century COE "Center for Diversity and Universality in Physics" of Kyoto University.

\section{References}

[1] H. Abuki and T. Kunihiro, Nucl. Phys. A 768 (2006) 118.

[2] M. Kitazawa, T. Koide, T. Kunihiro and Y. Nemoto, Phys. Rev. D 65, 091504 (2002).

[3] M. Kitazawa, T. Koide, T. Kunihiro and Y. Nemoto, Phys. Rev. D 70, 056003 (2004).

[4] M. Kitazawa, T. Koide, T. Kunihiro and Y. Nemoto, Prog. Theor. Phys. 114, 117 (2005).

[5] I. Giannakis and H. c. Ren, Nucl. Phys. B 669, 462 (2003).

[6] M. Matsuzaki, Phys. Rev. D 62, 017501 (2000).

[7] H. Abuki, T. Hatsuda and K. Itakura, Phys. Rev. D 65 (2002) 074014.

[8] see, for example, L.D. Landau and E.M. Lifshiz, Statistical Physics (Pergamon, New York, 1958).

[9] D.N. Voskresensky, Phys. Rev. C 69, 065209 (2004).

[10] Y. Yanase, T. Jujo, T. Nomura, H. Ikeda, T. Hotta and K. Yamada, Phys. Rep. 387, 1 (2003).

[11] As a experimental review, T. Timusk and B. Statt, Rep. Progr. Phys. 62, 61 (1999).

[12] M. Kitazawa, T. Kunihiro and Y. Nemoto, Phys. Lett. B 631, 157 (2005).

[13] L.G. Aslamazov and A.I. Larkin, Sov. Phys. Solid State 10, 875 (1968); K. Maki, Prog. Theor. Phys. 40, 193 (1968); R.S. Thompson, Phys. Rev. B1, 327 (1970).

[14] See for example, M. Le Bellac, Thermal Field Theory (Cambridge University Press, Cambridge, England 1996).

[15] M. Kitazawa and T. Kunihiro, unpublished. A preliminary result is presented at the JPS meeting at Kochi University, Sep.,2004.

[16] P. Jaikumar, R. Rapp and I. Zahed, Phys. Rev. C 65, 055205 (2002).

[17] T. Hatsuda and T. Kunihiro, Phys. Lett. 145, 7 (1984); Prog. Theor. Phys. 75, 765 (1985); Phys. Rev. Lett. 55, 158 (1985); Phys. Rep. 247, 221 (1994).

[18] F. Karsch, Lect. Notes in Physics, 583, 209 (2002).

[19] M. Gyulassy and L. McLerran, Nucl. Phys. A 750 (2005) 30. 
[20] M. Asakawa and T. Hatsuda, Phys. Rev. Lett. 92, 012001 (2004); S. Datta, F. Karsch, P. Petreczky and I. Wetzorke, Phys. Rev. D 69, 094507 (2004); T. Umeda, K. Nomura and H. Matsufuru, Eur. Phys. J. C 39S1, 9 (2005);

[21] M. Asakawa and K. Yazaki, Nucl. Phys. A504, 668 (1989);

M. Lutz, S. Klimt and W. Weise, Phys. Lett. B249, 386 (1990); Nucl. Phys. A542, 521 (1992).

[22] T. Kunihiro, Phys. Lett. B 219, 363 (1989); B245, 687 (1990)(E); Nucl. Phys. B351, 593 (1991).

[23] M. Stephanov, Prog. Theor. Phys. Suppl.153,139 (2004).

[24] M.Kitazawa, T. Koide, T. Kunihiro and Y. Nemoto, Prog. Theor. Phys. 108, 929 (2002).

[25] T. Hatsuda, M. Tachibana, N. Yamamoto and G. Baym, Phys. Rev. Lett. 97 (2006) 122001.

[26] H. Fujii, Phys. Rev. D67 (2003) 094018; H. Fujii and M. Ohtani, Phys. Rev. D70 (2004) 014016.

[27] D. T. Son and M. Stephanov, Phys. Rev. D70 (2004)056001.

[28] Y. Nemoto, M. Kitazawa and T. Kunihiro, in preparation.

[29] T. Kunihiro, Phys. Lett. B271, 395 (1991). 